\title{
The Clinical Test for the Sensory Interaction of Balance
}

\author{
Vicky S Khattar, Bachi T Hathiram
}

\begin{abstract}
The balance system requires the interaction of appropriate sensory inputs, a sound processor and coordinated outputs. The human body relies primarily on three important sensory inputs, i.e. somatosensory, visual and vestibular. Not only do these inputs provide vital information as regards body orientation and position but also interact with each other so as to provide a balanced fodder for the central nervous system to process.

Situations arise in clinical practice wherein patients lack one or more of these inputs, and need to excessively rely on the others for maintaining balance. Such patients may be able to ordinarily maintain their sense of balance during daily activities, and only when exposed to adverse or stressful situations, do they need to practice caution; and then, there are some who do not even have the luxury of managing their daily activities with ease.

It is vital for the therapist to assess and identify the weaknesses of a patient as regards the sensory inputs and help them to capitalise on their strengths.

The clinical test for the sensory integration of balance is a simple test that has been devised to easily and rapidly assess the dependence of a patient on various inputs and devise a rehabilitative strategy customized to each patient.

The testalso has other applications besides simply assessing balance inputs and they have been briefly described.
\end{abstract}

Keywords: Clinical test, Balance, Computerized dynamic posturography (CDP) test, Sensory organizational test (SOT), Motor control test (MCT), Clinical test for the sensory interaction on balance (CTSIB).

How to cite this article: Khattar VS, Hathiram BT. The C linical Test for the Sensory Interaction of Balance. IntJ Otorhinolaryngol Clin 2012;4(1):41-45.

\section{Source of support $\mathrm{Nil}$}

Conflict of interest: None declared

\section{INTRODUCTION}

The computerized dynamic posturography (CDP) test is an important component of the test battery that may be applied to patients with balance disorders. It comprises of two components: The sensory organisational test (SOT) and the motor control test (MCT).

The M CT assesses the motor processes that coordinate the action of leg and trunk muscles into discrete synergies that minimise sway and maintain the body's center of mass within its base of support. ${ }^{1,2}$

The sensory organization test (SOT) component of the CDP is specifically designed to assess the dependence of an individual on their visual, somatosensory and vestibular inputs. The equipment for the test is expensive and available usually in teaching institutions, or research facilities dedicated to vestibular and bal ance analysis. The limited availability of the equipment makes it 'out of reach' for a majority of patients and their treating clinicians. Besides thus being unable to include it in their diagnostic armamentarium, most clinicians are unable to standardise and compare their results; the vast number of patients suffering from balance disorders only compounds the problem.

It is to be understood that the central nervous system usually relies on one of the three abovementioned inputs for the information of orientation and, in normal healthy adults, it is the somatosensory inputs which are primarily relied upon. In case of inadequate input/inability of one input to provide the necessary information, the other inputs take over to overcome this inadequacy. Finally, in cases of any conflict of input, the central nervous system relies on the vestibular system to decide the orientation of the body. ${ }^{3}$ It is thus important to assess the relative dependability of an individual suffering from a balance disorder on various inputs to identify the deficits and take preventive measures to rehabilitate the patient into an imbalance-free state.

Citing the above theories of Nashner, in 1986, Shumway-Cook and Horak gave a simple example of a person standing in front of a moving bus; in this situation, while the visual input would suggest a relative motion between the person and the bus, the somatosensory inouts would suggest that the person is indeed stationery. The central nervous system would then rely on the vestibular system to determine the 'true' body position and having done so and concluded that there is no movement (as proposed by the somatosensory input) would accordingly NOT generate any equilibrium response. In their landmark article, they further went on to describe a simple test which would be able to test the same components as in the SOT (vide supra), they called it the clinical test for the sensory interaction on balance (CTSIB).

The ease with which the CTSIB could be devised coupled with its minimal costs, would ensure an assessment of the same components as the SOT simultaneously make it available at all centers and eventually hel $p$ in comparison of results in a standardized manner (Figs 1 to 6).

\section{TECHNIQUE}

This test uses two conflicting somatosensory and three visual conflicting inputs to simulate the six modules of the SOT. 


\begin{tabular}{llll}
\hline & & & \\
\hline & Eyes open & Eyes closed & Wearing the visual dome \\
\hline Standing on & Situation 1: & Situation 2: & Situation 3: \\
terra firma & Eyes open, & Eyes closed, & Wearing the visual dome, \\
& standing on & standing on standing on terra firma \\
& terra firma & terra firma & \\
Standing on & Situation 4: Situation 5: Situation 6: \\
foam & Eyes open, Eyes closed, Wearing the visual dome, \\
& standing on standing on standing on foam \\
& foam & foam & \\
\hline
\end{tabular}

Blindfold: The blindfold can either be a simple piece of cloth tied around the eyes or even the soft eye patches provided during air travel to block out the light. Either ways, it should be able to effectively block out any visual cues for the patient.

F oam: The foam is usually a medium density foam. There has been a lot of discrepancy on the thickness of the foam used, but the basic understanding is that the patient's feet should not be able to touch the ground so as to derive any somatosensory input from it. In published literature,

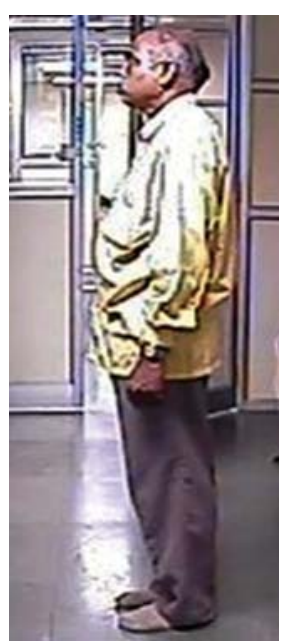

Fig. 1: Situation 1

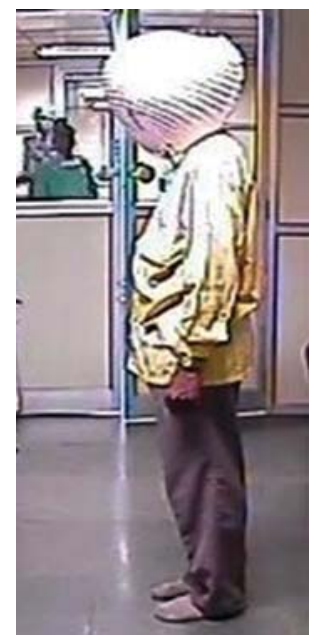

Fig. 3: Situation 3

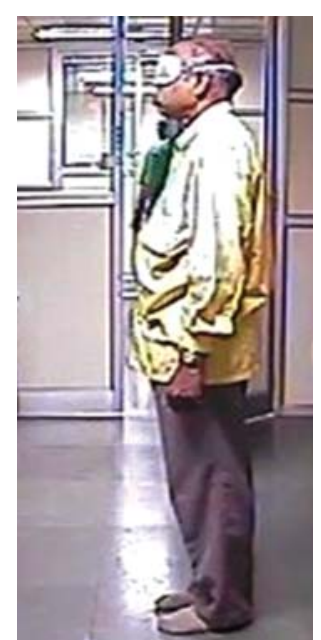

Fig. 2: Situation 2

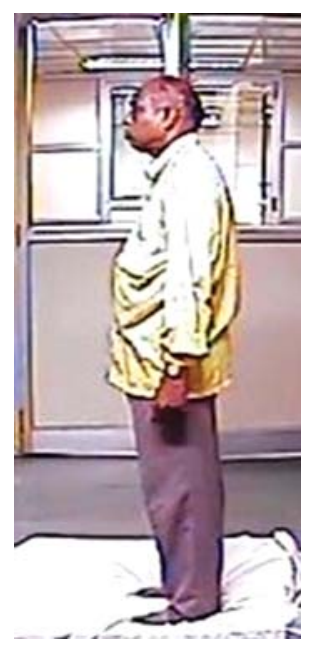

Fig. 4: Situation 4

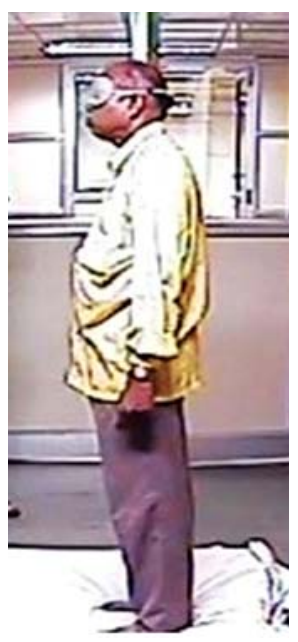

Fig. 5: Situation 5

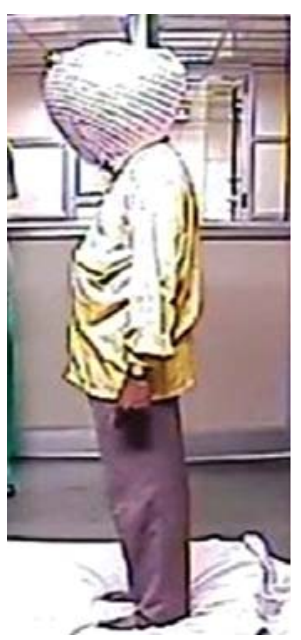

Fig. 6: Situation 6 thicknesses ranging from 3 inches to 7 inches have been described. The authors have been using a four inch medium density foam, that has been sufficient to test the various conditions with ease. In order to validate the test and remove the chances of any false positives, the authors have tested the normative responses of 'healthy' individuals, namely those without any balance disorder or complaint, and have found them to be able to sustain their balance with ease while standing in the mentioned foam, with eyes open and closed.

Dome: The classical description of Shumway-Cook and Horak (1986) used a Chinese lantern to devise the dome, and the authors have found the same to be the easiest and most inexpensive way to devise an 'effective' dome for testing. The lantern is 'cut out' of $90^{\circ}$ of its circumference to allow for the head of the patient to fit in. The frame of the lantern is used to mount it onto the frame of a headmirror which is routinely used for ENT examinations in the outpatient clinic. The inner aspect of the dome is lined with black insulation tape, in the form of lines that run down from the top to the bottom. Once fitted, the dome provides a 'sway referenced' visual input, wherein the visual stimulus moves in phase with the head of the patient. It is essential to block out all surrounding visual stimuli, in order to be able to truly test the response of the individual when the vision is sway referenced (Figs 7 to 9).

\section{Recording of Results}

Time: The time allocated for each situation should be atleast 30 seconds. In addition, the clinician may use a stopwatch to record the exact time that the patient is able to maintain balance (in case where the time is less than 30 seconds).

Stance: The patient should maintain the same stance in all six modules, to make the results comparable. Some authors 


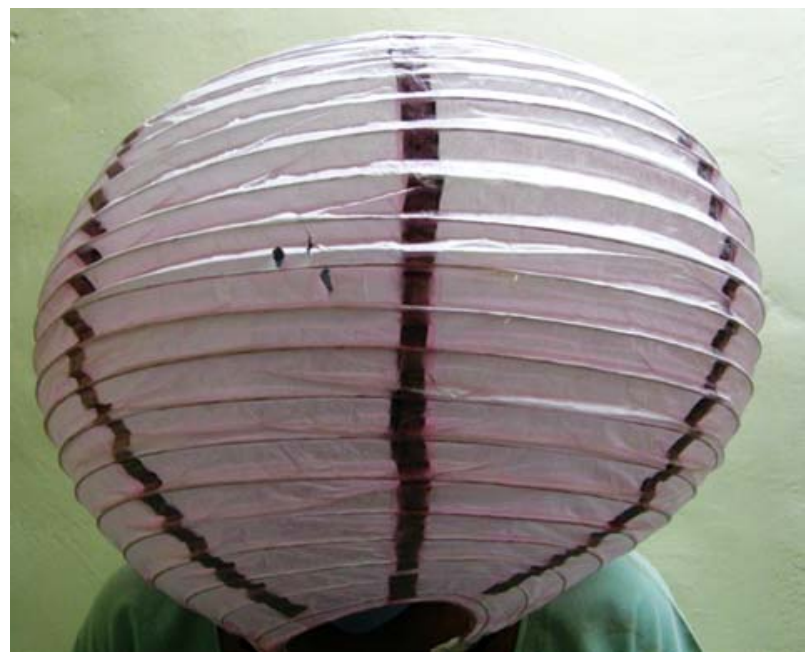

Fig. 7: The dome as seen from outside

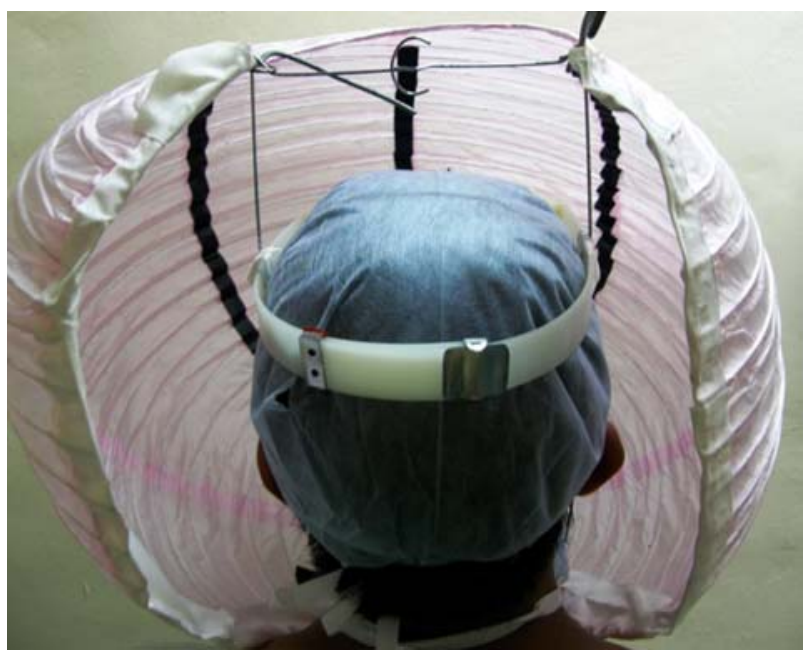

Fig. 8: The dome as seen from the outside after being worn by the patient

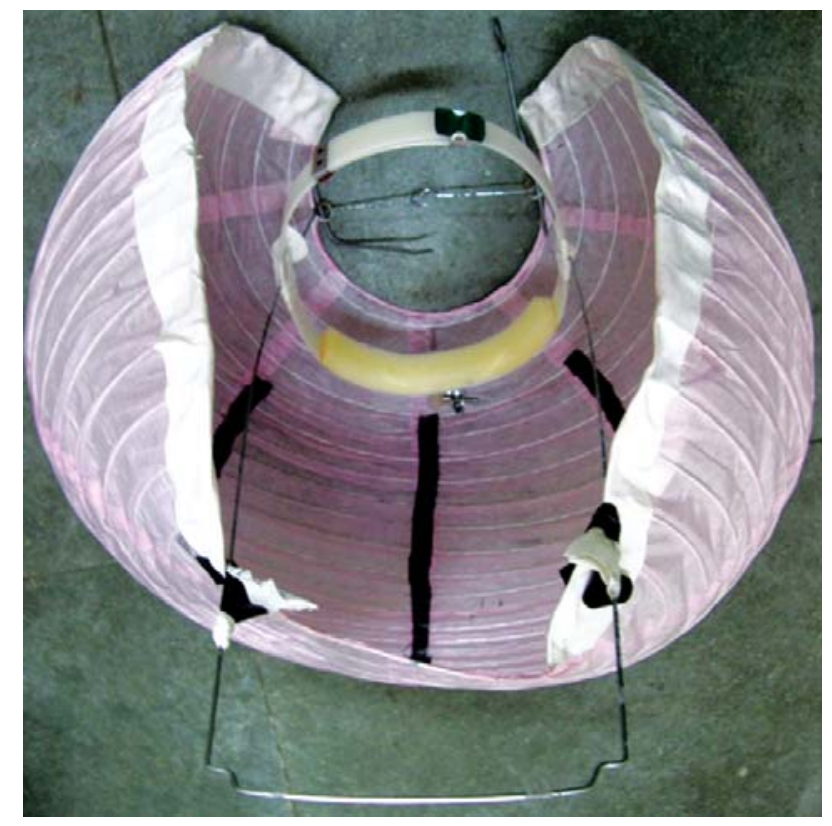

Fig. 9: The interior of the dome as seen. Note the frame of the dome mounted onto the band of the head-mirror and fixed with surgical tape. Also, note the vertical lines running from the top to bottom of the dome
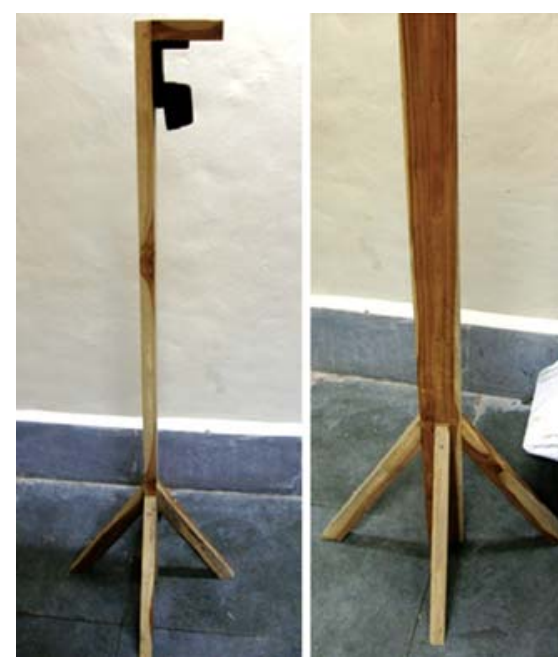

Fig. 10: To the left, note the simple wooden stand constructed to mount a CCTV camera (the black camera frame is seen) which will serve as the 'side' camera. To the right, note the base of the wooden stand that makes it extremely stable. The camera frame is at an approximate height of five feet

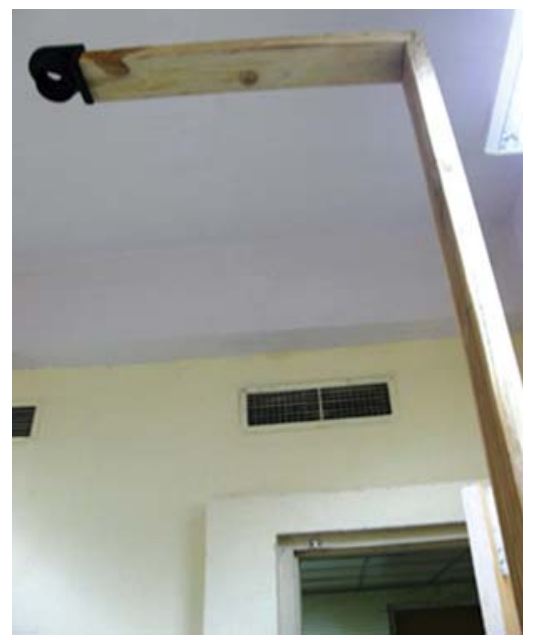

Fig. 11: The wooden frame for the 'top' camera, which reaches a height of eight feet and takes the patient's images from above. Thus using a combination of the 'side' and 'top' cameras, one can easily determine the orientation of the patient in both horizontal and vertical planes. The CCTV cameras used for this purpose are easily available and also have a 'night-vision' facility for patients who are not comfortable with the blindfold, and would rather prefer to stand in darkness to simulate the 'eyes-closed/blindfold' module

advocate the 'feet together' stance, while others advocate the 'feet apart' stance. $Y$ et others propose that the arms be crossed across the chest.

Visible sway: The sway may be semiquantified into a subjective rating of:

$$
\begin{aligned}
& 1=\text { minimal sway } \\
& 2=\text { mild sway } \\
& 3=\text { moderate sway } \\
& 4=\text { fall }
\end{aligned}
$$

by the clinician for documenting and comparison purposes. 
Subjective feeling by patient: The patient's reaction to the situation should also be recorded in the patients own words (and hence an audiovisual recording is preferable).

Adaptation strategy: This should also be recorded, so as to provide information to the physical therapist as regards the treatment strategy which may be best suited to the patients needs (Figs 10 and 11).

\section{Interpretation of Results}

A few factors must be kept in mind before interpreting the results.

F oam thickness: As mentioned earlier, the thickness of the foam used has been a topic of much debate. A variety of publications show the experience of authors with foams of different thicknesses, densities and age. In two of the frequently compared and published series of $W$ eber and Cass and EI-K ashlan, ${ }^{6,7}$ the foams used were 4 and 6 inches of medium density t-foam, and 4 inches of upholstery foam respectively. In contrast, the foam used by W risley and Whitney (2004) was a 3 inch high-density viscoelastic foam. Thus, until the foam densities are not standardized, it would be difficult to compare the results of the various series. The authors have used a 4 inch medium density foam for their tests. The only common factor is that in each series, was that no patient complained of their feet reaching the ground while standing on the foam, and till there is no consensus on the density and thickness of the foam, this may be used as a practical standard for testing.

A nother factor is the age of the foam, as suggested by W risley and Whitney (2004). As the foam ages, it tends to become firm, and this may alter the results of the test. Thus once again, it would be wise to standardize this factor along with the thickness and density as mentioned above.

Stance of the patient: W risley and Whitney (2004) have also discussed the foot position of the patient (feet apart vis a vis feet together) and found that the results of the test closely matched those of the formal SOT, when performed with the feet together. They explained this by the probability of the test becoming more difficult with the feet together, as when the feet are apart, they tend to move in different planes (due to the foam), and this would compound the results. Also, since it is in any case difficult for a patient to stand with feet together as compared to the feet apart, this test would automatically become more sensitive in unmasking balance problems. El-K ashlan tried to eliminate this problem of stance by asking the patient to stand on a cafeteria tray beneath the feet, which was in turn placed on foam.

\section{DISCUSSION}

In order to be able to design a treatment strategy for a balance patient, it is vital to identify the sensory input that the patient relies on primarily as well as his/her reaction and eventual dependence on the various sensory inputs during times of intersensory conflict. ${ }^{4}$

In a study by Crotts et al (1996), ${ }^{5}$ they compared the balance abilities of dancers versus nondancers using this 'foam and dome' test, and found significant differences between the two. Besides this being a unique use of this test, it makes one understand that the strategies used by dancers to train may be a useful adjunct to rehabilitate balance patients, with the CTSIB test as an objective/ semiquantitative parameter by which they may be evaluated for progress and eventual recovery.

A nother useful application of this test is to evaluate the adult population in a community, and identify the prospective 'fallers' among the elderly people. ${ }^{8}$ This would help in identifying those at risk, and taking the necessary preventive and rehabilitative measures to prevent these elders from falling and their associated comorbidities.

In spite of the various issues regarding standardization, this test has proved to be useful for assessing the balance function in various scenarios. A dded to that, the simplicity of constructing the apparatus, and the low cost would surely make it an easy alternative to the SOT.

\section{CONCLUSION}

The CTSIB besides being an effective, cheap and valid alternative to the more formal SOT, has numerous applications as mentioned above. In addition, it is an important component of the armamentarium of the balance therapist to identify the deficiencies in the patients' sensory integration, and take the appropriate corrective rehabilitative measures. It is easy to construct and maintain. The existing shortcoming lies in the validation of the equipment and standardization of certain components which would make it easier for multicentric data and analysis to be done. Nevertheless, it gives a reasonably good estimate of the parameters that are assessed and can by themselves prove to be valuable.

\section{REFERENCES}

1. N ashner LM . A dapting reflexes controlling the human posture. Exp B rain Res 1976;26:59-72.

2. Horak FB, Nashner LM. Central programming of postural movements: Adaptation to altered support surface configurations. J Neurophysiol 1986;55:1369-81. 
3. Nashner LM. A daptation of human movement to altered environments. Trends in Neuroscience 1982;5:351-61.

4. Shumway-CookA, Horak FB. A ssessing theinfluence of sensory interaction on balance. Phys Ther 1986;66(10):1548-50.

5. Crotts $D$, Thomson B, N ahom M, Ryan S, N ewton RA. B alance abilities of professional dancers on select balance tests. J ournal of Orthopaedic and Sports Physical Therapy 1996;23(1):12-17.

6. Weber PC, Cass SP. Clinical assessment of postural stability. Am J Otol 1993;14:566-69.

7. El-K ashlan HK, Shepard NT, A sher A M , Smith-Wheelock M, Telian SA. Evaluation of clinical measures of equilibrium. Laryngoscope 1998;108:311-19.

8. Desai A, Goodman V, Kapadia N, et al. Relationship between dynamic balance measures and functional performance in community dwell ling el derly people. Phys Ther 2010;90:748-60.

\section{ABOUT THE AUTHORS}

\section{Vicky S Khattar}

A ssistant Professor, D epartment of ENT and Head and N eck Surgery Topiwala N ational M edical Collegeand BY L N air Charitable H ospital M umbai, M aharashtra, India

Correspondence Address: C-7, Swati K alyan Complex Panch M arg Off Y ari Road, V ersova, M umbai-4000061 M aharashtra, India, Phone: 91-9930977110, 91-9324655600 e-mail:vickykhattar@rediffmail.com,orlclinics@gmail.com

\section{Bachi T Hathiram}

Professor and H ead, D epartment of ENT and Head and Neck Surgery Topiwala $\mathrm{N}$ ational M edical Collegeand BY L N air Charitable H ospital M umbai, M aharashtra, India 